\title{
Reseña de Viganoni, Lida (Ed.). (2019). Commercio e consumo nelle città che cambiano: Napoli, città medie, spazi esterni. Milán: Franco Angeli.
}

David Lloberas LAFUENTE

Universitat de Barcelona

España

\section{Contexto y líneas generales}

El volumen Commercio e consumo nelle città che cambiano constituye la contribución de la Universitá de Napoli "L'Orientale", en colaboración con la Universitat de Barcelona, al Progetto di ricerca di Rilevante Interesse Nazionale (PRIN), financiado por el Ministerio Italiano de Educación, Universidades e Investigación. La profesora Lida Viganoni, del Departamento de Ciencias Humanas y Sociales de "L'Orientale", ha sido la editora de este volumen y la encargada de coordinar el proyecto entre las universidades de Barcelona y las universidades de Bologna, Chieti-Pescara, Mascerata (Messina), Politecnico di Milano, Palermo y Trieste.

Bajo el título Commercio, consumo e città: pratiche, pianificazione e governance per l'inclusione, la resilienza e la sostenibilità urbane, este proyecto iniciado en 2017 está materializado en siete volúmenes, uno por cada universidad, junto con un cuaderno de trabajo. El proyecto se centra, por su relevancia estratégica, en la evolución de las relaciones que existen en el consumo y el comercio con la ciudad, y en el carácter multidimensional y multiescalar de los fenómenos que caracterizan los espacios urbanos en la actualidad.

El presente volumen, Comercio y consumo en ciudades cambiantes: Nápoles, ciudades medianas, espacios al aire libre, por su título en español, continúa con las líneas de investigación desarrolladas a través de la red internacional Ciudad, Comercio y Consumo, en la que, además de Nápoles y Barcelona, participan grupos de investigación de larga trayectoria radicados en diferentes universidades de México, Brasil, Argentina y Portugal. Estas miradas desde el mundo latino y mediterráneo contribuyen en el estudio de las relaciones históricas y contemporáneas del comercio y el consumo en la ciudad, con una mirada crítica desde fuera de las áreas centrales del mundo anglosajón, cuya literatura sobre el tema es muy relevante (Gasca y Olivera, 2017: 12; Montesinos Ciuró, 2019: 213). En el seno de esta red ya se han celebrado siete seminarios 
internacionales, siendo los más recientes en Ciudad de México (2015), Lisboa (2017) y Buenos Aires (2019). ${ }^{1}$

El presente libro reseñado tiene la finalidad de proveer nuevas indicaciones para incidir en políticas urbanas en favor de la inclusión social, la resiliencia urbano-comercial y la sostenibilidad. En su conjunto, el volumen realiza investigaciones sobre la lógica y el alcance de las transformaciones de los espacios urbanos al mismo tiempo que se analizan los diversos fenómenos sociales y culturales que las acompañan. Como parte de un proyecto de relevancia estratégica que es este volumen, se expresa con gran con claridad el funcionamiento actual de los complejos urbano-comerciales considerando su planificación integrada a la ciudad, ante la enorme profusión de estas actividades, especialmente en el campo del comercio minorista, que están modelando las ciudades italianas y europeas.

\section{Contenido y estructura}

A través de un marco teórico-metodológico rico en conceptos de naturaleza diversa, se analizan temas relacionados con la estructura urbana, los conflictos entre agentes urbanos y los diversos procesos urbanos articulados por las tipologías comerciales — por ejemplo, las relaciones centro-periferia, el uso del espacio público, los "centros comerciales naturales", la sustitución comercial, las estrategias de localización, los procesos de gentrificación residencial y comercial, entre otros-. Para desarrollar estos temas, el volumen aborda la investigación empírica en tres fases, que en sí misma constituye una gran contribución al caracterizar las formas, agentes y perfiles de comerciantes y consumidores. En primer lugar, aborda la observación directa de los lugares a ser estudiados, seguida de la creación de material censal a través de una clasificación tipológica y el consecuente cartografiado de su organización espacial. Finalmente, se aborda la realización de entrevistas destinadas a diferentes agentes comerciantes y consumidores, que ocasionalmente también son residentes de las áreas de estudio. La realización de estas entrevistas se adaptó a las características de las áreas comerciales, clasificadas en tres categorías: calles comerciales, centros comerciales/outlets y mercados. Los destinatarios de las preguntas - comerciantes y consumidores - se orientaron para indagar sobre temas diferentes: forma de negocio, motivación de la localización, tipo de clientela, opiniones sobre las políticas comerciales, por un lado; y motivación de la elección del sitio, horarios, medios de transporte utilizado, procedencia, por otro. De las 716 y 875 entrevistas a comerciantes y consumidores respectivamente, 502 y 636 se efectuaron en las ocho calles comerciales, 83 y 88 en los dos centros comerciales, y 131 y 133 en los cuatro mercados objeto de estudio.

${ }^{1}$ Las memorias del $7^{\circ}$ Seminario se encuentran en proceso de publicación. 
La estructura general del libro consiste en cuatro partes, además de una introducción y las conclusiones: i) Marco teórico, ii) Realidades urbanas paralelas, iii) Fuera de Nápoles, y iv) Dentro de Nápoles. El primer capítulo de la primera parte, escrito por Carles Carreras y Sergi Martínez-Rigol, de la Universitat de Barcelona, y titulado "Los grandes cambios en la sociedad de los consumidores y sus consecuencias conceptuales", trata los diferentes cambios acontecidos a nivel político, económico, tecnológico y social en el mundo a partir de la referencia de dos eventos históricos traumáticos: la crisis de los años 70 y la recesión que estalló en 2008. Asimismo, se discuten los conceptos clave para el desarrollo de este libro, como mercado, comercio, clasificación comercial, proximidad, atracción comercial y empresa.

El segundo capítulo de la primera parte, escrito por Rosario Somella y titulado "Il territorio della ricerca: fuori e dentro Napoli”, delimita las áreas de estudio dentro y fuera de Nápoles, donde aquellas áreas localizadas en la Campania metropolitana (Marcianise y Aversa) son tratadas como puntos en el espacio, mientras que aquellas que son internas a la ciudad se estudian como líneas o ejes, o puntos en el caso de los mercados. Por otro lado, el tercer capítulo, escrito por Libera D'Alessandro y titulado "Le politiche per il commercio: scale, tempi, strumenti", es un capítulo dedicado al análisis de los méritos y límites de las diferentes herramientas regulativas y de las políticas urbano-comerciales, en un contexto de la liberalización del sector y de la coexistencia de multitud de marcos normativos dictados desde instancias europeas, estatales, regionales y municipales.

En el primer capítulo de la segunda parte, "Consumo y comercio en Barcelona. Actualidad y tendencias", escrito por Carles Carreras, Lluís Frago Clols, Eduard Montesinos y Alejandro Morcuende, se aborda la continuidad de la política comercial municipal, retratando la actual estructura comercial de Barcelona bajo la aproximación a la división social y territorial del trabajo desde la economía política, la reconversión de los antiguos espacios industriales en espacios comerciales y, finalmente, se evalúa el impacto del consumo y el comercio a escala metropolitana y regional. El capítulo "Profitto e valori: ethos e commercio. Il caso di Matera", escrito por Ornella Albolino, Lucia Cappiello, Giovanna Iacovone y Luigi Stanzione, de la Università degli Studi della Basilicata, trata principalmente de los impactos socioeconómicos de un turismo en crecimiento exponencial y las relaciones territoriales que mantienen con el área metropolitana de Bari. De acuerdo con la editora del volumen, el caso de Matera es "el emblema de una ciudad media del Mezzogiorno italiano que emerge vigorosamente de un largo sueño" (Viganoni, 2017: 19).

Ya en el contexto metropolitano, en la tercera parte del libro, "Fuori Napoli", se estudian las áreas intersticiales; su primer capítulo, "Attrattività e problematiche nell'area di Marcianise”, escrito por Libera D'Alessandro, trata del llamado “consumo experiencial" que se materializa en alternativas a las posibilidades de consumo que ofrecen los centros urbanos, concretamente en un centro comercial y un outlet de nueva generación. En cuanto al segundo capítulo, escrito por la editora del volumen Lida Viganoni, "Commercio e città medie: il caso di Aversa”, se analiza tanto el papel 
del centro histórico de Aversa como "centro comercial natural", no sólo por su peso desde el punto de visa poblacional, sino a causa de su autonomía a nivel funcional respecto a la ciudad vecina de Caserta.

En la cuarta parte, "Dentro Napoli", se tratan los efectos de la turistificación y la foodizzazione que afectan algunas áreas del centro histórico, y que reconfiguran las formas de comercio y las prácticas de consumo. El primer capítulo, escrito por los arquitectos Bruno Discepolo e Irene Lettieri y titulado "L'asse commerciale di via Chiaia: storia ed evoluzione", se centra en las transformaciones a nivel urbanístico y en la erosión de los espacios residenciales en Via Chiaia y alrededores; mientras que en el segundo, titulado "Via Chiaia tra forme di sostituzione del commercio e nuove pratiche di appropriazione dello spazio", D'Alessandro destaca la mayor intensidad en la transformación de los espacios públicos ligada a la proximidad de la Luxury Shopping Line (LSL). Por su parte, la Vía Toledo es abordada por Libera D'Alessandro en “Microgeografie di un'icona simbolica del commercio napoletano: via Toledo tra mutamenti e contese", enfocada a la gran fuerza de atracción de esta localización para marcas dedicadas al fast fashion y actividades similares. Y desde un punto de vista antropológico, Flavia Cuturi y Marzia Mauritello en "Scambi di patrimoni: itinerari etnografici tra i cibi di strada a via Toledo" pone el centro de atención en los procesos históricoculturales que de acuerdo con las autoras ha conducido esta calle a una situación de "reinvención de la tradición".

Viganoni y D'Alessandro, en "La Luxury Shopping Line tra commercio e movida", destacan la representatividad de lo que denominan como "nueva teatralidad" del comercio en espacios urbanos híbridos en sus funciones. Por otro lado, "La 'svolta' verso cibo e turismo: via dei Tribunali e via Benedetto Croce", escrito por estas dos profesoras con Annachiara Autiero, representa una contribución para la "interpretación de las diferentes etapas que han conducido a la actual configuración comercial", con una fuerte presencia de cadenas de comida rápida. Por su parte, Fabiana Sciarelli, Roberto Vona y Fabio Greco en "L'evoluzione della distribuzione alimentare e dei consumi: gli ethnic food e i superfood", investigan los cambios en oferta y demanda de productos alimentarios de origen extranjero de carácter multi-étnico. Rosario Somella y Eleonora Guadagno, en "Spazi e tempi del commercio su aree pubbliche", a través de encuestas realizada en cuatro mercados, analizan la diversificación de las funciones de estos espacios terciarizados y revelan la centralidad de la actividad comercial sobre el conjunto de la ciudad.

El presente volumen trata con gran profundidad la configuración actual del comercio y el consumo en Nápoles y su área metropolitana, y lo hace de una manera integrada y en diálogo en relación con las dinámicas continentales y globales. La editora italiana Franco Angeli ha puesto a disposición esta colección de siete libros con acceso abierto para el público especializado. Ésta no sólo constituye una obra de gran relevancia académica y técnica que, siendo rica en teoría e indagación de campo, permite vislumbrar los cambios y tendencias de un periodo que constituye una crisis en sí mismo (Santos, 2000). 
$82 \square$ RESEÑAS

\section{Referencias bibliográficas}

Gasca, José; Olivera, Patricia (Coords.). (2017). Ciudad, comercio urbano y consumo: Experiencias desde Latinoamérica y Europa. Ciudad de México: Instituto de Investigaciones Económicas, UNAM.

Montesinos Ciuró, Eduard. (2019). “D’Alessandro, Libera (Ed.). (2015). City, retail and consumption". Tlalli. Revista de Investigación en Geografia, (1), 213-218. https://doi.org/10.22201/ffyl.26832275e.2019.1.394

Santos, Milton. (2000). Por uma outra globalização: do pensamento único à consciência universal. Río de Janeiro: Record.

Viganoni, Lida (Ed.). (2017). Commercio, consumo e città. Quaderno di lavoro. Milán: Franco Angeli. 